\title{
Association of Infant and Child Feeding Index with Undernutrition in Children Aged 6-59 Months: A Cross-Sectional Study in the Maldives
}

\author{
ljaz ul Haq, ${ }^{1,2 \star} †$ Mariyam Asra, ${ }^{2,3} \dagger$ Qing Tian, ${ }^{1}$ Bilal Ahmed, ${ }^{4}$ Nadar Khan, ${ }^{5}$ Muhammad ljaz Ahmad, ${ }^{6}$ Chenming Ji, ${ }^{1}$ \\ and Jianguang Luo ${ }^{1}$ \\ ${ }^{1}$ School of Food Science, Jiangsu Food \& Pharmaceutical Science College, Huai'an, Jiangsu, China; ${ }^{2}$ Department of Nutrition and Food Hygiene, \\ Nanjing Medical University, Nanjing, China; ${ }^{3}$ Department of Food Control Division, Maldives Food and Drug Authority, Ministry of Health, Malé, \\ Maldives; ${ }^{4}$ Department of Clinical Pharmacology, School of Pharmacy, Nanjing Medical University, Nanjing, China; ${ }^{5}$ Department of Animal \\ Nutrition, The University of Agriculture, Peshawar, Pakistan; ${ }^{6}$ College of Food Science and Technology, Nanjing Agricultural University, \\ Nanjing, China
}

\begin{abstract}
Adequate dietary intake is critically important for child growth and development. This study aimed to analyze the prevalence of undernutrition and its association with infant and child feeding index (ICFI). This cross-sectional study was conducted among children (younger than5 years) and their mothers from Lhaviyani Atoll, Maldives. The data were obtained by interviewing the children's mothers via pretested questionnaires. Infant and child feeding index scores were calculated from the dietary information. Weight-for-age z-scores (WAZ), length/height-for-age z-scores (LAZ/HAZ), and weight-forlength/height $z$-scores were calculated from anthropometric data taken according to the WHO criterion. Linear regression tests were used to find the association of nutritional status with ICFI scores. A total of 800 children and their mothers participated in this study. The prevalence of underweight, stunting, and wasting was $24.6 \%, 32.4 \%$, and $16.3 \%$, respectively. The mean ICFI scores (13.0) of children aged 6-8 months were better than those of children in other age-groups. In food groups, the intake of fish was higher among the respondents, whereas the consumption of vegetables and fruits was lower. Infant and child feeding index scores were significantly associated $(P<0.05)$ with WAZ and LAZ/HAZ after adjustment for confounders. Overall, the findings showed that Maldivian children consumed the limited number of food items that resulted in an inadequate intake of nutrients which further resulted in the high prevalence of malnutrition.
\end{abstract}

\section{INTRODUCTION}

Child undernutrition is a major public health concern for developing countries. ${ }^{1}$ About $45 \%$ of deaths in children younger than 5 years are due to nutrition-related factors. ${ }^{2}$ According to the United Nations Children's Fund (UNICEF), in 2018, globally, 149 million children younger than 5 years are having stunting, and 49.5 million are having wasting. In Asia, these numbers are higher in the Southern Asian region, where the prevalence of wasting and stunting are $14.6 \%(25.3$ million) and $32.7 \%$ (57.9 million), respectively. ${ }^{3}$ In the Maldives, according to the UNICEF, in 2013, the prevalence of underweight, stunting, and wasting was $26 \%, 32 \%$, and $13 \%$, respectively. ${ }^{4}$

Early childhood undernutrition predicts the risk of disease in later life. ${ }^{5}$ Proper and adequate nutrition is necessary for the development of the child at an early age. Poor feeding practices and inadequate dietary practices in infants and children, along with infectious diseases, are the causes of child undernutrition. ${ }^{6,7}$ Moreover, feeding practices are associated with physical development, and improper feeding practices lead to poor physical development in infants and young children. ${ }^{8}$ Consuming fewer food groups high in protein and calcium like milk products and poultry/meat may be a causing factor for child malnutrition, especially stunting. ${ }^{9}$

Estimation of proper dietary intake and child feeding practices is necessary to minimize the risks of undernutrition in children. To estimate optimal feeding practices, in the

\footnotetext{
*Address correspondence to ljaz ul Haq, School of Food Science, Jiangsu Food and Pharmaceutical Science College, No. 4 Meicheng Road, Huai'an, Jiangsu 223003, China, E-mail: ijazbrt@outlook.com or Mariyam Asra, Department of Food Control Division, Maldives Food and Drug Authority, Ministry of Health, Malé 20379, Maldives, E-mail: mariyamasra1987@gmail.com.

†These authors contributed equally to this work.
}

literature, different child feeding indicators and indices have been reported. One of these indices is ICFI (infant and child feeding index), made in 2002, and included information on breastfeeding, dietary diversity, intake of food rich in nutrients, and frequency of meal. ${ }^{10}$ Infant and child feeding index can be used to measure the infant and young child feeding practices. ${ }^{11}$ This index has been associated with parameters of malnutrition in children. In rural western China, ICFI scores were associated with overall length, weight, weight-for-age Z-score (WAZ), and HAZ quantiles. ${ }^{8}$ In India, children having higher ICFI scores had better WHZ, WAZ, and HAZ. ${ }^{11}$

Undernutrition is a serious public health issue in the Maldives. Although the Maldivian government has already achieved five of the eight Millennium Development Goals, including the goal of reducing the number of people suffering from hunger, still the prevalence of stunting and underweight is high. ${ }^{12}$ The key determinants of undernutrition in this region are high disease burden, poor infant feeding practices, and limited access to nutritious food. ${ }^{13}$ Because of the importance of child dietary intake and feeding, it is necessary to accurately estimate the infant and child feeding practices and associate it with the undernutrition outcomes in this population. We used ICFI scores to estimate the feeding practices in children younger than 5 years. The current study aimed to evaluate the association between ICFI and undernutrition in children younger than 5 years in Lhaviyani Atoll islands, Maldives. This study will help the policymakers in Maldives to develop and improve contextual interventional measures, and will also address and solve the influencing factors of undernutrition.

\section{METHODS}

Study population. A cross-sectional study among children aged 6-59 months and their mothers from Lhaviyani Atoll local islands (Naifaru, Hinnavaru, Kurendhu and Olhuvelifushi) was 
conducted through two-stage cluster sampling techniques. The sample size was calculated according to the standard formula $n=714$ children plus $12 \%$ (86 children) to cater for nonresponse. ${ }^{14}$ Therefore, the total sample size was 800 children.

Inclusion and exclusion criteria. Only mothers with children younger than 5 years and willing to participate in the study were included in this study. All the respondents were residents of Lhayiyani Atoll Naifaru, Hinnavaru, Kurendhu, and Olhuvelifushi for at least 6 months before the study.

Children older than 5 years suffering from chronic illnesses or having no consent from the parents to participate in the study were excluded. The health condition of the children was verified using their health cards.

Data collection. The questionnaire was first designed in the English language, and later, it was translated to Dhivehi language to make it easy for the participants to understand the questions. Data were collected via face-to-face interviews during the growth monitoring visits and household visits. The study was conducted from June 1, 2017 to November 30, 2017 with the help of 20 trained interviewers.

Information on children's date of birth, gender, exclusive breastfeeding, weight/height or length, illness history, as well as household demographics and socioeconomic status were collected through interviews with mothers. Before each interview, the purpose of the survey was explained to the mother and written informed consent was obtained.

Anthropometry and other information. Anthropometric data were included by weight, height for those who could stand, and length for those who could not. Z-score was calculated to find out the weight-for-length/height $z$-score (WLZWHZ), WAZ, and length/height-for-age $z$-score (LAZ/HAZ) according to the WHO criterion. ${ }^{11,15}$ Other reported details included hospital visits, child's medical history, active sleeping history, growth assessment, history of breastfeeding, eating environment, food safety, drinking habits, and food frequency.

Infant and child feeding index scores. Infant and child feeding index scoring was constructed according to the previous studies. ${ }^{10,16}$ During the interview, information on the local feeding practices was collected, including breastfeeding, bottle-feeding, and seven-day food-group frequency questionnaire. Seven food groups used to estimate dietary intake: 1) dairy products, 2) carbohydrate-rich foods, 3) meat and fish, 4) egg, 5) vegetable protein, 6) vitamin A-rich fruits and vegetables, and 7) other vegetables/fruits. Participants were asked about the consumption of food groups consumed by the child. For the consumption of each food group, a score ranging from 0 to 2 was given. If a food group was not consumed, then the score was 0 ; if consumed for 1-3 days, then the score was 1; and if consumed for 4 days or more, then the score was 2 . All the scores for different food groups were summed to have a total score ranging from 0 to 29 . Based on this total score, a new score of 0-2 was assigned, reflecting the age-specific distribution. For example, in the case of a child belonging to the age-group 6-8 months, a total score of 0 was also considered 0 under the new score. The total score ranging from 1 to 2 was considered 1 . The total score of 3 or more was considered two under the new score of food-group frequency. The detail of the scoring system specific for each of the three age-groups is summarized in Supplemental Table 1. For each age-group, the ICFI score ranged from 0 to 19 .

Statistical analysis. EpiData 3.1 (The Epidata Association, Odense, Denmark), WHO Anthro 3.2.2 (WHO, Geneva, Switzerland), and SPSS 21 (SPSS, Chicago, IL) were used for further analysis. Primary study outcomes were the rates of malnutrition based on WLZ/WHZ, WAZ, and LAZ/HAZ. Secondary outcomes included infant and child feeding information. Descriptive statistics, like frequency and percentage, were used. Linear regressions were used to find the association of ICFI scores with malnutrition parameters. Our linear regression models were valid as there was no issue of heteroskedasticity. The plot of residuals showed an even envelope of residuals created in the linear regression plots for all models. Moreover, the tolerance score was more than 0.1 , and the variance inflation factor (VIF score) was between 1 and 10 for all models that showed no issue of multicollinearity. ${ }^{17} P<$ 0.05 was considered statistically significant.

\section{RESULTS}

General characteristics of the study population. A total of 800 mothers and their children (boys $=399$ and girls $=401$ ) were included in the survey (Table 1). The majority of mothers (95.9\%) were married, and $64.5 \%$ of mothers had completed secondary education, followed by $19.9 \%$ who had a university education. Regarding occupation, the majority of the respondents $(53.4 \%)$ were housewives. The family income of

TABLE 1

General characteristics of the study population

\begin{tabular}{|c|c|c|}
\hline Variable & Mean \pm SD or $N$ & Percentage \\
\hline \multicolumn{3}{|l|}{ Gender } \\
\hline Male & 399 & 50.0 \\
\hline Female & 401 & 50.0 \\
\hline \multicolumn{3}{|l|}{ Age-group (months) } \\
\hline $6-8$ & 25 & 9.1 \\
\hline $9-11$ & 48 & 25.4 \\
\hline $12-36$ & $\mathrm{~s}$ & 25.4 \\
\hline $37-59$ & 320 & 41.0 \\
\hline \multicolumn{3}{|l|}{ Mothers' educational level } \\
\hline Primary education & 116 & 1.5 \\
\hline Secondary education & 516 & 64.5 \\
\hline College & 159 & 19.9 \\
\hline Illiterate & 9 & 1.1 \\
\hline \multicolumn{3}{|l|}{ Mothers' marital status } \\
\hline Married & 767 & 95.8 \\
\hline Divorced & 33 & 4.2 \\
\hline \multicolumn{3}{|l|}{ Mothers' occupation } \\
\hline Government job & 76 & 9.5 \\
\hline Self-employed & 154 & 19.3 \\
\hline Private job & 143 & 17.9 \\
\hline Others (housewife) & 427 & 53.4 \\
\hline \multicolumn{3}{|l|}{ Family income, MVR } \\
\hline Less than 4,000 & 9 & 1.1 \\
\hline $4,000-6,000$ & 101 & 12.6 \\
\hline Greater than 6,000 & 690 & 86.3 \\
\hline \multicolumn{3}{|l|}{ Household size } \\
\hline Less than 3 & 32 & 4 \\
\hline Between 3 and 6 & 399 & 49.9 \\
\hline More than 6 & 369 & 46.1 \\
\hline \multicolumn{3}{|c|}{ Number of children younger than 5 years in the family } \\
\hline Less than 3 & 718 & 89.8 \\
\hline Between 3 and 6 & 61 & 7.6 \\
\hline More than 6 & 21 & 2.6 \\
\hline \multicolumn{3}{|l|}{ Malnutrition } \\
\hline Underweight & -0.74 & 1.2 \\
\hline Stunting & -0.89 & 1.4 \\
\hline Wasting & -0.38 & 1.3 \\
\hline
\end{tabular}




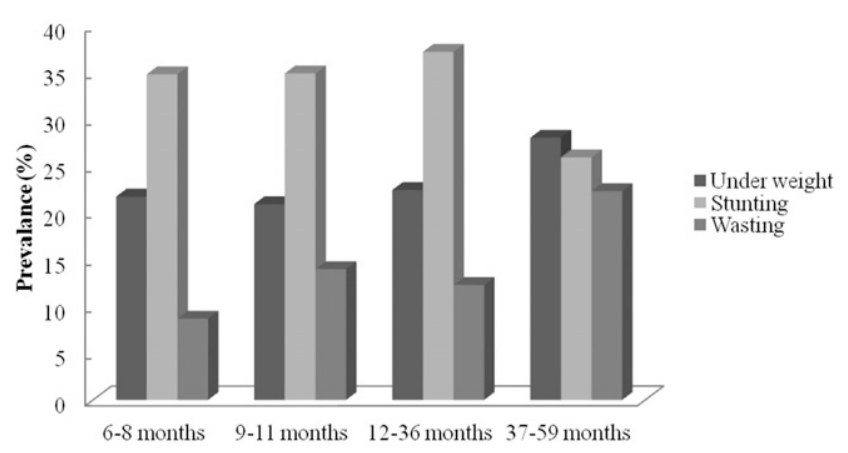

FIGURE 1. Prevalence of malnutrition (underweight, stunting, and wasting) in the children according to age.

$86.3 \%$ of the participants was more than 6,000 Maldivian Rufiyaa per month. The majority of the houses $(89.8 \%)$ had less than three children younger than 5 years.

Prevalence of malnutrition. The prevalence of malnutrition in children younger than 5 years in terms of stunting, wasting, and underweight has been described in Supplemental Table 2. It was found that $32.4 \%$ of the children had low LAZ/HAZ (stunted), 16.3\% had low WLZ/WHZ (wasted), and $24.6 \%$ had low WAZ (underweight).

Figure 1 describes the prevalence of malnutrition according to age. It was found that $22.4 \%$ of children in the age-group 12-36 months and $28.0 \%$ of children in the age-group 3759 months were underweight. As for stunting, the prevalence was $37.2 \%$ in the children of the age-group 12-36 months. Wasting $(22.3 \%)$ was higher in the children of the age-group 37-59 months.

Feeding practices of the children. Table 2 shows that $36 \%$ of the children were breastfed and $48 \%$ bottle-fed at the time of the interview. It was found that $84.9 \%$ (aged 68 months) used one food group, whereas $57.1 \%$ (aged 911 months) used one to two food groups and $50.0 \%$ (aged 12-36 months) used two or three food groups of a dairy product. The children of 6-8 months (67.1\%) age-group used carbohydrate-rich one or two food groups, and of $37-59$ months (55.3\%) age-group used seven or more food groups weekly. The highest of children, 88.2\% (aged 911 months), and the lowest of children, 82.2\% (aged 68 months), used five food groups of fish. The majority of children, 52.1\% (aged 6-8 months), used three or more food groups of other vegetables and fruits, whereas $58.1 \%$ (aged 37-59 months) used five or more food groups weekly. Almost every age-group child was fed three or more times (44.8\%; aged 9-11 months), four or more times (42.6\%; aged 12-36 months, and 42.0\%; aged 3-59 months); however, $37 \%$ (children aged 6-8 months) were fed two or more times. The mean ICFI score was 13 for children aged 68 months, 11.6 for chidren aged 9-11 months, 12.2 for chidren aged 12-36 months, and 10.6 for chidren aged 37-59 months.

Association of ICFI with nutritional status. The association of ICFI scores and malnutrition parameters WLZMHZ, WAZ, and LAZ/HAZ has been shown in Table 3. In the multivariate models, ICFI scores were having independent associations $(P<0.05)$ with WAZ, and LAZ/HAZ after adjustment for child age, gender, maternal education, family size, and family income.

\section{DISCUSSION}

The current study investigated dietary intake, feeding practices, nutritional status, prevalence of undernutrition, and association of ICFI scores with undernutrition in 6- to 59-month-old Maldivian children. Infant and child feeding index scores were significantly associated with stunting and underweight in the Maldives. Furthermore, the prevalence of stunting and wasting was higher in people aged 37-59 years. Up to our best knowledge, the current study is the first study that describes the association of malnutrition with ICFI scores in the Maldives.

The prevalence of underweight, stunting, and wasting in the current study was found to be $24.6 \%, 32.4 \%$, and $16.3 \%$, respectively. These findings were similar to that of report shared by the UNICEF where $26 \%$ of children younger than five years are underweight, $32 \%$ stunted, and $13 \%$ wasted. ${ }^{4}$

TABLE 2

Feeding practices of study children

\begin{tabular}{|c|c|c|c|c|c|c|c|}
\hline ICFI Component & & $\begin{array}{l}6-8 \text { months } \\
(n=25), \%\end{array}$ & & $\begin{array}{c}9-11 \text { months } \\
(n=48), \%\end{array}$ & & $\begin{array}{c}12-36 \text { months } \\
(n=407), \%\end{array}$ & $\begin{array}{c}37-59 \text { months } \\
(n=320), \%\end{array}$ \\
\hline Breastfeeding & Yes & 91.8 & Yes & 65.3 & Yes & 38.2 & 4.4 \\
\hline Bottle-feeding & Yes & 47.9 & Yes & 53.7 & Yes & 54.7 & 39.7 \\
\hline \multicolumn{8}{|l|}{ Received a food group previous 7 days } \\
\hline \multirow[t]{2}{*}{ Dairy product } & 1 & 84.9 & $1-2$ & 57.1 & 2 or 3 & 50.0 & 36.4 \\
\hline & 2 or more & 6.8 & 3 or more & 10.8 & 4 or more & 12.3 & 12.9 \\
\hline \multirow[t]{2}{*}{ Carbohydrate-rich food } & 1 or 2 & 67.1 & $2-4$ & 35.1 & $4-6$ & 44.6 & 26.9 \\
\hline & 3 or more & 28.8 & 5 or more & 18.7 & 7 or more & 29.4 & 55.3 \\
\hline \multirow{2}{*}{ Fish } & $3-4$ & 2.7 & 4 & 3.9 & 4 & 2.5 & 5.3 \\
\hline & $\geq 5$ & 82.2 & $\geq 5$ & 88.2 & $\geq 5$ & 86.3 & 88.1 \\
\hline \multirow[t]{2}{*}{ Egg } & $3-4$ & 13.7 & $4 \mathrm{Fg}=1$ & 12.4 & $4=1$ & 18.1 & 30.9 \\
\hline & $\geq 5$ & 42.5 & $\geq 5 \mathrm{Fg}=2$ & 45.5 & $\geq 5$ & 59.3 & 54.7 \\
\hline \multirow[t]{2}{*}{ Vitamin A-rich vegetables and fruits } & 1 or 2 & 19.2 & $2-4$ & 23.6 & $2-4$ & 27.9 & 37.2 \\
\hline & 3 or more & 28.8 & 5 or more & 27.1 & 5 or more & 41.7 & 30.0 \\
\hline \multirow[t]{2}{*}{ Other vegetables and fruits } & 1 or 2 & 20.5 & $2-4$ & 28.6 & $2-4$ & 23.5 & 25.9 \\
\hline & 3 or more & 52.1 & 5 or more & 14.5 & 5 or more & 55.4 & 58.1 \\
\hline \multirow[t]{3}{*}{ Feeding frequency } & Once $=1$ & 39.7 & 1 or $2=1$ & 38.4 & 3 times $=2$ & 42.2 & 51.1 \\
\hline & 2 or more $=2$ & 37.0 & 3 or more $=2$ & 44.8 & 4 or more $=3$ & 42.6 & 42.0 \\
\hline & & $13.0(1.8)$ & & $11.6(2.6)$ & & $12.2(2.8)$ & $10.6(2.1)$ \\
\hline
\end{tabular}

$\mathrm{ICFI}=$ infant and child feeding index. 
TABLE 3

Association of ICFI with child nutritional status

\begin{tabular}{|c|c|c|}
\hline & $\beta$ & $P$-value \\
\hline Relation with weight-for-length/height z-score ${ }^{a}$ & & \\
\hline ICFI & 0.069 & 0.335 \\
\hline Relation with weight-for-age $z$-score ${ }^{b}$ & & \\
\hline ICFI & 0.162 & 0.015 \\
\hline Relation with length/height-for-age $z$-score ${ }^{c}$ & & \\
\hline ICFI & 0.233 & 0.003 \\
\hline
\end{tabular}

monthly income, family size, and maternal education.

These figures reflect that over a period of time, the condition of malnutrition is on the same lines.

One of the factors contributing to malnutrition in children younger than 5 years in the Maldives is dietary intake and feeding practices. Earlier, a study reported their undernutrition status as $37.7 \%$ underweight, $18.2 \%$ wasted, and $36.8 \%$ stunted, and all the respondents ate starchy foods, $61 \%$ ate fish, and $76 \%$ ate milk and milk products. ${ }^{18}$ In the current study, children of different age-groups consumed starchy foods, milk products, and fish intake more than five times a week. Most of the children consumed a variety of foods containing carbohydrates, animal protein, and a few varieties of vegetables and leaves in their daily dietary intake. Previously in the Maldives, children aged 1-4 years have the same or slightly lower intake of micronutrient. Their diets contain enough carbohydrates and protein but lacked fats. ${ }^{19}$

Individual food may not appropriately predict the nutritional status of children. Therefore, consuming a variety of food groups combined in a specific sequence and scoring might appropriately predict the nutritional status of children younger than 5 years in the Maldives. We used ICFI scores. Previously, ICFI scores have been used to find the proper dietary intake and feeding practices, and have been associated with the nutritional status of children according to the context. $^{10,20}$

Our findings showed that there was an independent positive association between WAZ and ICFI. Similarly, LAZ/HAZ was also having an independent positive association with child age (months) after adjustment of confounders. In India, Burkina Faso, and parts of Latin America, it was found that the ICFI was significantly associated with HAZ and WAZ. ${ }^{21,22}$ Earlier, it has also shown that inadequate dietary habits and feeding practices are the proximate causes of undernutrition in children. ${ }^{6}$ According to the UNICEF, undernutrition, including underweight, stunting, and wasting, is the outcome of insufficient dietary intake and infectious diseases. ${ }^{23}$

There were certain limitations to this study. The study was a cross-sectional design. The current study used quantitative data. The questionnaire did not contain any question which has to be answered in detail. In addition, some information may be biased because the results were obtained according to the answers of the respondents. Moreover, this study is not generalized to the whole population of the Maldives because this research only focused one region.

\section{CONCLUSION}

In conclusion, the prevalence of undernutrition in the Maldives is still high, which could lead to child morbidity and mortality. Maldivian children consumed a limited number of food items that resulted in an inadequate intake of nutrients which resulted in the high prevalence of malnutrition in the region. The overall calculated scores for ICFI was fair but needs further improvement to decrease undernutrition.

Based on these findings, community-based interventions need to be communicated and implemented to improve child health. At an individual level, interventions should focus on educating mothers regarding the basics of proper nutrition and the need to use available health services, whereas on a community level, healthcare systems that facilitate public health interventions such as maternal and child health programs need to be made accessible to women in local islands. These interventions will improve the nutritional status of children younger than 5 years in the Maldives. Children during the early years of age undergo rapid growth and development that is greatly influenced by the aforementioned factors. Exclusive breastfeeding, adequate complementary feeding, a safe environment, and care need to be ensured for optimum physical, mental, social, and cognitive development. In addition, it could prevent adverse impacts on short-term survival as well as long-term health and development.

In future, longitudinal studies need to confirm the associations between ICFI scores and malnutrition. Also, future studies regarding dietary patterns and child health may be helpful in improving the region's malnutrition burden.

Received December 31, 2019. Accepted for publication April 14, 2020. Published online May 18, 2020.

Note: Supplemental tables appear at www.ajtmh.org.

Acknowledgment: We are very thankful to Ahmed Sajid (Manager, Health services Lh. Atol Hospital Maldives) for his kind support in this study.

Disclosure: I. H. and M. A. designed and wrote the original draft; M. A. collected the data; B. A., N. K., and C. J. contributed to editing and formal analysis; and M. I. A., L. J., and Q. T. reviewed the article. This study was conducted according to the guidelines laid down in the Declaration of Helsinki, and all procedures involving research study participants were approved by the Ethical Committee of Nanjing Medical University. Written informed consent was obtained from all subjects.

Authors' addresses: ljaz ul Haq, Qing Tian, Chenming Ji, and Jianguang Luo, Department of Food Science, Jiangsu Food Science College, Huaian, China, E-mails: ijazbrt@outlook.com, 376541542@ qq.com, jcm0609@163.com, and 20101003@jsfpc.edu.cn. Department of Food Control Division, Maldives Food and Drug Authority, Ministry of Health, Malé, Maldives, E-mail: mariyamasra1987@ gmail.com. Bilal Ahmed, Department of Clinical Pharmacology, School of Pharmacy, Nanjing Medical University, Nanjing, P. R. China, E-mail: drbilal_71@yahoo.com. Nadar Khan, Department of Animal Nutrition, The University of Agriculture, Peshawar, Pakistan, E-mail: nadar73vet@aup.edu.pk. Muhammad ljaz Ahmad, College of Food Science and Technology, Nanjing Agricultural University, Nanjing, China, E-mail: ejazifst@gmail.com.

\section{REFERENCES}

1. Dhami MV, Ogbo FA, Osuagwu UL, Agho KE, 2019. Prevalence and factors associated with complementary feeding practices among children aged 6-23 months in India: a regional analysis. BMC Public Health 19: 1034

2. Khanam M, Shimul SN, Sarker AR, 2019. Individual-, household-, and community-level determinants of childhood undernutrition in Bangladesh. Health Serv Res Manag Epidemiol 6: 2333392819876555.

3. United Nations Children's Fund (UNICEF), World Health Organization, International Bank for Reconstruction and Development/The 
World Bank, 2019. Levels and Trends in Child Malnutrition: Key Findings of the 2019 Edition of the Joint Child Malnutrition Estimates. Geneva, Switzerland: World Health Organization.

4. World Bank Group, 2013. World Development Indicators 2013. Washington, DC: World Bank Group.

5. Biro FM, Wien M, 2010. Childhood obesity and adult morbidities. Am J Clin Nutr 91: 1499S-1505S.

6. Custodio E, Herrador Z, Nkunzimana T, Weziak-Bialowolska D, Perez-Hoyos A, Kayitakire F, 2019. Children's dietary diversity and related factors in Rwanda and Burundi: a multilevel analysis using 2010 demographic and health surveys. PLoS One 14: e0223237.

7. Joe $\mathrm{W}$ et al., 2019. Association between anthropometric-based and food-based nutritional failure among children in India, 2015. Matern Child Nutr 15: e12830.

8. Qu P, Mi B, Wang D, Zhang R, Yang J, Liu D, Dang S, Yan H, 2017. Association between the infant and child feeding index (ICFI) and nutritional status of 6- to 35-month-old children in rural western China. PLoS One 12: e0171984.

9. Mahmudiono T, Sumarmi S, Rosenkranz RR, 2017. Household dietary diversity and child stunting in East Java, Indonesia. Asia Pac J Clin Nutr 26: 317-325.

10. Moursi MM, Treche S, Martin-Prevel Y, Maire B, Delpeuch F, 2009. Association of a summary index of child feeding with diet quality and growth of 6-23 months children in urban Madagascar. Eur J Clin Nutr 63: 718-724.

11. Chaudhary SR, Govil S, Lala MK, Yagnik HB, 2018. Infant and young child feeding index and its association with nutritional status: a cross-sectional study of urban slums of Ahmedabad. $J$ Fam Community Med 25: 88-94.

12. UNICEF, 2012. Available at: https://www.unicef.org/nutrition/ maldives_62471.html. Accessed May 17, 2012.

13. World Bank, 2013. Maldives - Nutrition at a Glance (English). Nutrition at a Glance; Maldives. Washington DC: World Bank.

14. SurveyMonkey, 2018. Sample Size calculator. Available at: https://www.surveymonkey.com/mp/sample-size-calculator/. Accessed January 5, 2018.
15. Wirth JP et al., 2019. Growth status, inflammation, and enteropathy in young children in northern Tanzania. Am J Trop Med Hyg 100: 192-201.

16. Ruel MT, Menon $P, 2002$. Child feeding practices are associated with child nutritional status in Latin America: innovative uses of the demographic and health surveys. J Nutr 132: 1180-1187.

17. Haq IU et al., 2019. Association of smoking-related knowledge, attitude, and practices (KAP) with nutritional status and diet quality: a cro4-sectional study in China. Biomed Res Int 2019: 5897478.

18. Ali NB, Tahsina T, Hoque DME, Hasan MM, Iqbal A, Huda TM, El Arifeen S, 2019. Association of food security and other socioeconomic factors with dietary diversity and nutritional statuses of children aged 6-59 months in rural Bangladesh. PLoS One 14: e0221929.

19. Golder AM, Erhardt JG, Scherbaum V, Saeed M, Biesalski HK, Furst $P, 2001$. Dietary intake and nutritional status of women and pre-school children in the republic of the Maldives. Public Health Nutr 4: 773-780.

20. Moursi MM, Martin-Prevel Y, Eymard-Duvernay S, Capon G, Treche S, Maire B, Delpeuch F, 2008. Assessment of child feeding practices using a summary index: stability over time and association with child growth in urban Madagascar. Am J Clin Nutr 87: 1472-1479.

21. Lohia N, Udipi SA, 2014. Infant and child feeding index reflects feeding practices, nutritional status of urban slum children. BMC Pediatr 14: 290.

22. Sawadogo PS, Martin-Prevel $Y$, Savy M, Kameli $Y$, Traissac $P$, Traore AS, Delpeuch F, 2006. An infant and child feeding index is associated with the nutritional status of 6- to 23-month-old children in rural Burkina Faso. J Nutr 136: 656-663.

23. Abdulahi A, Shab-Bidar S, Rezaei S, Djafarian K, 2017. Nutritional status of under five children in Ethiopia: a systematic review and meta-analysis. Ethiop J Health Sci 27: 175-188. 